\title{
Why Are The Secondary Stars in Polars So Normal?
}

\author{
Thomas E. Harrison \\ Department of Astronomy New Mexico State University, Box 30001, MSC 4500, Las \\ Cruces, NM 88003-8001 \\ Steve B. Howell \\ WIYN Observatory and National Optical Astronomy Observatories, 950 North Cherry \\ Avenue, Tucson, AZ 85726 \\ Paula Szkody \\ Department of Astronomy, University of Washington, Box 351580, Seattle, WA 98195 \\ and \\ France A. Cordova \\ Institute of Geophysics and Planetary Physics, Department of Physics, University of \\ California, Riverside, CA 92521 \\ Key words: infrared: stars — cataclysmic variables
}

Received __; accepted _ 


\begin{abstract}
We have used NIRSPEC on Keck II to obtain $K$-band spectroscopy of several magnetic cataclysmic variables. These data reveal that the secondary stars in these binary systems have spectra that are consistent with normal, late-type dwarfs in both their atomic and molecular line strengths, as well as in the slopes of their continuua. This result is in stark contrast to the infrared spectra of their non-magnetic cousins, nearly all of which show peculiar abundances, especially of CNO species and their isotopes. It appears that the evolutionary path taken by the secondary stars in magnetic systems differs from that for the non-magnetic systems. We discuss the implications of this result.
\end{abstract}




\section{Introduction}

Cataclysmic variables (CVs) are short-period binary systems consisting of a white dwarf primary that is accreting material via Roche-lobe overflow from a low mass, late-type secondary star. CVs can be divided into two main classes: magnetic and non-magnetic. In magnetic systems, the primary white dwarf has a strong magnetic field (0.5 MG $\leq \mathrm{B} \leq 240 \mathrm{MG})$. In "Polars", the magnetic field of the white dwarf is strong enough to capture the accretion stream close to the secondary star. In "Intermediate Polars" (IPs), the magnetic field of the white dwarf is believed to be weaker $(\mathrm{B} \leq 8 \mathrm{MG})$, and is insufficient to completely prevent the formation of an accretion disk. IPs therefore exhibit behavior that can be unique, or common to both magnetic and non-magnetic classes.

The commonly proposed evolutionary history for cataclysmic variables has been assumed to be similar for both the magnetic and non-magnetic systems (King et al. 1994, Kolb 1995), and has three main phases. First, the orbital separation of the wide binary of the pre-CV is rapidly shrunk in a common envelope phase where the secondary star orbits inside the red giant photosphere of the white dwarf progenitor. The second phase is a very long epoch where gravitational radiation, or a magnetically constrained wind from the secondary star extracts angular momentum from the binary (magnetic braking), resulting in the eventual contact of the photosphere of the secondary star with its Roche lobe. The final phase begins once the secondary star contacts its Roche lobe, mass transfer to the white dwarf is initiated, and all of the phenomena associated with CVs is observed.

Based on the current paradigm (see Howell, Nelson, \& Rappaport 2001, and references therein) it can be argued that the majority of secondary stars in CVs should show little signs of evolution at the time of contact - the duration 
of the CV formation process is a small fraction of a low mass star's main sequence lifetime. In addition, after contact, the secondary star begins to lose mass, extending its lifetime, and preventing it from ever forming a He-burning core (Howell 2001). Recent results, however, challenge this idea. Infrared spectroscopy of two dozen CVs by Harrison et al. (2004, 2005), and UV spectroscopy (Gänsicke et al. 2003, and references therein) find evidence for peculiar abundance ratios in the secondary stars of non-magnetic CVs. The dominant anomalies are deficits of carbon and enhancements of nitrogen. In addition, several CVs show evidence for enhanced levels of ${ }^{13} \mathrm{CO}$ in their $K$-band spectra. Taken together, these results suggest that CNO-processed material has found its way into the photospheres of CV secondary stars. Either the accretion of material during the common envelope phase and/or from classical novae eruptions is much more efficient than expected, or the secondary stars in non-magnetic CV systems started out with much larger masses (to allow CNO burning) than current population synthesis theories predict. If magnetic systems followed the same evolutionary path prior to the contact phase, then they too should have secondary stars with similar abundance anomalies. In the following we present new infrared $K$-band spectroscopy for five polars: VV Pup, ST LMi, AR UMa, MR Ser and SDSS J1553+5516.

\section{Observations}

Infrared spectroscopy for the program objects was obtained using NIRSPEC ${ }^{1}$ on Keck II in photometric conditions on 17 February 2005. A journal of our observations is presented in Table 1. We used NIRSPEC in low resolution mode

\footnotetext{
${ }^{1}$ For more on NIRSPEC go to http://www2.keck.hawaii.edu/inst/nirspec/nirspec.html
} 
with a 0.38 " slit. The grating tilt was set so as to cover the wavelength region $2.04 \mu \mathrm{m} \leq \lambda \leq 2.46 \mu \mathrm{m}$, with a dispersion of $4.27 \AA$ pixel $^{-1}$. We employed the two-nod script, and we used four minute exposure times for all of the program CVs. To correct for telluric absorption, we observed bright A0V stars located close to the program objects so as to minimize their relative differences in airmass. These data were reduced using the IDL routine "redspec", specially developed for NIRSPEC ${ }^{2}$. In the $K$-band, the spectra of A0V stars are nearly featureless, except for the prominent H I Brackett $\gamma$ feature at $2.16 \mu \mathrm{m}$. The redspec package does not attempt to correct for this feature, but can interpolate across such lines to reduce their impact upon division into the program star spectrum. Note that there is a weak telluric feature located very close to the Brackett $\gamma$ line, and thus the $\mathrm{H}$ I line profiles in spectra produced by the division of a "patched" A-star spectrum are slightly compromised.

In Fig. 1 we present the final, medianed spectra of VV Pup, ST LMi, AR UMa, and SDSS J1553+5516. The NIRSPEC spectrum of MR Ser is shown in Fig. 2, where it is compared to a 2003 May spectrum obtained using SPEX on the IRTF. As seen in Table 1, the time spent on ST LMi and AR UMa was relatively short, $\leq 10 \%$ of an orbital period, and thus smearing of the secondary star features due to orbital motion is not significant. This is not the case for VV Pup, SDSS J1553+5516, and MR Ser. For MR Ser and VV Pup we have used published ephemerides by Schwope et al. (1993) and Walker (1965) to correct for the radial velocity motion of their secondary stars. A radial velocity analysis of the VV Pup data set presented here (Howell et al. 2005, in prep.), confirms the

\footnotetext{
${ }^{2}$ Details about the redspec package can be found here: http://www2.keck.hawaii.edu/inst/nirspec/redspec/index.html
} 
phasing from Walker (1965). No such ephermerides exist for SDSS J1553+5516, and thus the orbital motion of its secondary star cannot be accounted for. Fortunately, its spectrum does not show signs of significant smearing. The spectra in Fig. 1 have been smoothed to a resolution of $5.1 \AA$ pixel $^{-1}$ to allow us to directly compare them to spectra of late-type dwarfs obtained using SPEX on the IRTF (from Harrison et al. 2005). It is important to note that the redspec package flux calibrates the spectra using a blackbody spectrum while the SPEXTOOL package (Vacca et al. 2003) used to produce the late-type dwarf spectra from SPEX data employs a model A-star atmosphere. Any subtle differences in the slopes of the continuua between the program objects presented here, and the late-type templates observed with SPEX, could in fact be due to the slightly different flux calibration procedures used by the two packages, and not due to any intrinsic differences.

\section{Results}

Close examination of the spectra shown in Fig. 1 reveal no significant abundance anomalies (see Harrison et al. 2005 for identifications of the stronger spectral features found in late-type dwarfs). The atomic absorption lines and CO absorption features appear to have strengths that are consistent with those expected for late type dwarfs. We have derived spectral types for each of the secondary stars and list them in Table 1. The spectral features in VV Pup are somewhat broader and/or weaker than in the other program objects, suggesting a large rotational velocity, or improper phasing. VV Pup was also the faintest of the five polars, and thus its spectrum is somewhat noisier. Even with this, it is clear that the Na I doublet (near $2.20 \mu \mathrm{m}$ ) in VV Pup has a strength relative to the CO features (at $2.294 \mu \mathrm{m}$, and redward) that is consistent with it being 
a normal, very late M-type dwarf. As demonstrated by the weakness of their emission lines, all of the polars (except MR Ser) were in low states, a time when the accretion rate from the secondary star drops to a very low value, and the activity level of the system declines. As seen in Fig. 2, the emission lines from He I and H I in MR Ser were prominent, suggesting it was in an active state, though no visual estimates of the system brightness exist to confirm this conclusion. Due to this activity level, the spectrum of the secondary star in MR Ser is considerably diluted compared to that observed during a lower level of activity. During the low state, analysis of the IR spectrum of MR Ser obtained with SPEX on the IRTF lead to a classification of M8V. Thus, the secondary stars of these five polars, plus that of the proto-type system, AM Her (Harrison et al. 2005), appear to be relatively normal late-type dwarfs.

That the secondary stars of polars show no evidence for peculiar abundance patterns is in stark contrast to our results for non-magnetic systems (Harrison et al. 2004, 2005). In those efforts, only a single system (IP Peg) out of twenty CVs had a secondary star with normal CO absorption features. This suggests that the evolutionary histories of magnetic and non-magnetic systems are different.

Since all known CV secondary stars have masses below $1.3 \mathrm{M}_{\odot}$, generating peculiar abundances via the $\mathrm{CNO}$ cycle in $\mathrm{CV}$ secondary stars cannot occur after contact (Howell 2001). Thus, any observed abundance anomalies are either 1) the result of normal stellar evolution during the pre-CV phase, 2) are due to material of peculiar composition being accreted during the common envelope phase, or 3) comes from the accretion of classical novae ejecta once the mass transfer phase ensues (see Marks \& Sarna, 1998). Both magnetic (e.g., V1500 Cyg, Stockman et al. 1988; CP Pup, Diaz \& Steiner 1991; V2214 Oph, Baptista et al. 1993; V2487 Oph, Hernanz \& Gloria 2002; BT Mon, White et al. 1996; 
GQ Mus, Diaz \& Steiner 1994) and non-magnetic systems have been observed as classical novae. As shown in calculations by Livio, Shankar, \& Truran (1988), the field strengths found in polars (and expected in IPs) are insufficient to inhibit, or dramatically alter, classical novae outbursts. Thus, the accretion of novae ejecta as an explanation for unusual abundance patterns in CV secondary stars appears to be eliminated. It is critical, however, to show that one or more of the classical novae listed above are true polars, and are not simply IPs like the proto-type magnetic classical nova DQ Her.

Kolb (1995), King et al. (1994), and Leibert et al. (2005) all conclude that the presence of a highly magnetic "white dwarf" primary cannot strongly affect the common envelope phase of evolution unless the secondary star orbits close to the magnetospheric radius of the magnetic core. If true, we are left to conclude (under the standard CV evolutionary paradigm) that the abundance anomalies we detect in non-magnetic systems must be due to evolutionary effects in the secondary stars themselves. This implies that the secondary stars of non-magnetic systems must start life with a mass sufficient to initiate the CNO cycle $\left(\mathrm{M} \geq 1.3 \mathrm{M}_{\odot}\right)$ so as to enrich some layers within their atmospheres. The current, low mass secondary star is then the stripped remains of this more massive object.

If peculiar CNO abundances in non-magnetic CVs result from normal stellar evolution, then the secondary stars in polars must have entered and exited the pre-contact phases with masses similar to what is currently observed. Given the observed preference of polars to have shorter periods than non-magnetic systems (see King et al. 1994, and references therein), it is relevant to ask whether all $\mathrm{CVs}$ below the famous period gap are simply the product of pre-CV binaries with initially low mass secondary stars. The UV study by Gänsicke et al. (2003) 
shows that at least one, normal, non-magnetic system below the priod gap, BZ UMa, shows a large $\mathrm{N} \mathrm{V/C} \mathrm{IV} \mathrm{emission} \mathrm{line} \mathrm{ratio.} \mathrm{It} \mathrm{is} \mathrm{vital} \mathrm{to} \mathrm{attempt} \mathrm{to}$ obtain data on more CV systems below the period gap to investigate whether this is true for all short period non-magnetic systems.

A recent FUV survey of eleven polars by Araujo-Betancor et al. (2005) finds normal $\mathrm{N} \mathrm{V/C} \mathrm{IV} \mathrm{emission} \mathrm{line} \mathrm{ratios} \mathrm{for} \mathrm{several} \mathrm{systems,} \mathrm{indicating} \mathrm{that} \mathrm{the}$ material being transfered to the white dwarf in those polars is not of unusual composition, adding further weight to our results. We note, however, that the asynchronous, long period polar, BY Cam, has extreme N V/C IV line ratios (Mouchet et al. 2003), providing at least one, albeit peculiar, counter-example. The strong correspondence between the $\mathrm{N}$ V/C IV emission line ratios in the UV, and the strength of the secondary star's CO features in the IR, is remarkable, and it appears that one can be used as a proxy for the other.

We conclude that the evolutionary history of most polars appears to differ from that of the majority of non-magnetic CVs. While the exact origin for this difference could be the initial masses of their secondary stars, it is hard to understand why low mass binaries preferentially go on to produce magnetic white dwarf primaries. A related conundrum is the apparent lack of "pre-polars" (Schmidt et al. 2005, Wellhouse et al. 2005, and references therein), binary systems containing magnetic white dwarf primaries and main sequence secondary stars that are not in contact with their Roche lobes. Does the magnetism of the white dwarf primary accelerate the pre-contact evolution to the point that nearly all polars are born in their currently observed states? Schmidt et al. suggest that the small family of "Low Accretion Rate polars" (LARPs, Schwope et al. 2002), including SDSS J1553+5516, are in fact the pre-polars. However, EF Eri is currently behaving in a fashion that is similar 
to this group of LARPs, a condition attained only seven years after an observed high state (Harrison et al. 2004). Given the ability of EF Eri to get stuck in such a prolonged low state, it is probably too early to conclude that the recently discovered LARPs never have high states.

Our results for magnetic systems has shed new light on non-magnetic systems: It now seems that the most likely path for the extreme levels of carbon depletion found in non-magnetic systems is the pre-contact evolution of the secondary star. The secondary stars in most non-magnetic CVs must have started out life with much higher masses than is observed now, in direct conflict with population synthesis theories.

Given this scenario, it is interesting to postulate whether Algols might be the progenitors of CVs. Algols are plentiful, both components in most systems appear to have had initial masses high enough to ignite the CNO cycle during their lifetimes, and many Algols have orbital periods ( $\sim 1$ day) that fall in the range to make suitable pre-CV candidates. In addition, the stellar components in numerous Algol systems show peculiar abundances of CNO elements, including enhanced levels of nitrogen, and deficits of carbon (Cugier \& Hardorp 1988, Cugier 1989, Parthasarathy et al. 1983, Tomkin \& Lambert 1989). Two particularly relevant Algols, that already have total system masses similar to those of the longest period CVs, are TT Hya and S Cnc. Both TT Hya and S Cnc have B9.5V primaries, and cool late-type giant/subgiant secondary stars with exceptionally small masses of 0.4 and $0.2 \mathrm{M}_{\odot}$, respectively (Olson \& Etzel 1993; Etzel 1988). The existence of a "flip-flop", where the primary and secondary star switch roles, was long ago (Paczynski 1971) proposed to explain the presence of low mass, evolved secondary stars in Algols with high mass primaries. It might be time to explore the possibility that a subset of Algols 
are the progenitors of non-magnetic CVs, where the peculiar secondary stars in both are the remnant "cores" of the same, once more massive stars.

Acknowledgments: Data presented herein were obtained at the W.M. Keck Observatory, which is operated as a scientific partnership among the California Institute of Technology, the University of California and the National Aeronautics and Space Administration. The Observatory was made possible by the generous financial support of the W.M. Keck Foundation. The authors wish to recognize and acknowledge the very significant cultural role and reverence

that the summit of Mauna Kea has always had within the indigenous Hawaiian community. We are most fortunate to have the opportunity to conduct observations from this mountain.

\section{References}

Araujo-Betancor, S., Gänsicke, B. T., Lonk, K. S., Beuermann, K., de Martino, D., Sion, E. M., \& Szkody, P. 2005, ApJ, 622, 589

Baptista, R., Jablonski, F. J., Cieslinski, D., \& Steiner, J. E., 1993, ApJ, 406, L67

Cugier, H. 1989, A\&A, 214, 168

Cugier, H., \& Hardorp, J. 1988, A\&A, 202, 101

Diaz, M. P., \& Steiner, J. E. 1991, PASP, 103, 964

Diaz, M. P., \& Steiner, J. E. 1994, AJ, 425, 252

Etzel, P. B. 1988, AJ, 95, 1204

Gänsicke, B. T., Szkody, P., de Martino, D., Beuermann, K., Long, K. S., Sion, E. M., Knigge, C., Marsh, T., Hubeny, I. 2003, ApJ, 594, 443

Harrison, T. E., Osborne, H. L., \& Howell, S. B. 2005, AJ, 129, 2400

Harrison, T. E., Osborne, H. L., \& Howell, S. B. 2004, AJ, 127, 3943

Hernanz, M., \& Sala, G. 2002, Science, 298, 393 
Howell, S. B., Harrison, T. E., Szkody, P. 2004, ApJ, 602, L49

Howell, S. B., Nelson, L. A., Rappaport, S. 2001, ApJ, 550, 897

Howell, S. B. 2001, PASJ, 53, 675

King, A. R., Kolb, U., de Kool, M., \& Ritter, H. 1994, MNRAS, 269, 907

Kolb, U. 1995, in "Cape Workshop on Magnetic Cataclysmic Variables", ASP

Conf. Series, Vol 85, ed. D. A. H. Buckley and B. Warner (ASP: San

Francisco), p 440

Leibert, J., et al. 2005, AJ, 129, 2376

Livio, M., Shankar, A., \& Truran, J. W. 1988, ApJ, 330, 264

Marks, P. B., \& Sarna, M. J. 1998, MNRAS, 301, 699

Mouchet, M., et al. 2003, A\&A, 401, 1071

Olson, E. C., \& Etzel, P. B. 1993, AJ, 106, 1162

Paczynski, B. 1971, Ann. Rev. Astron. Astrophys. 9, 183

Parthasarathy, M., Lambert, D. L., \& Tomkin, J. 1983, MNRAS, 203, 1063

Schmidt, G. D., et al. 2005, ApJ in press, astro-ph/0505385

Schwope, A. D., Beuermann, K., Jordan, S., \& Thomas, H. -C. 1993, MNRAS

278,487

Schwope, A. D., Brunner, H., Hambaryan, V., Schwarz, R., Staude, A., Szokoly, G., \& Hagen, H.-J. 2002, in "The Physics of Catclysmic Variables and Related Objects", ASP Conf. Ser. 261, ed. Gänsicke, B. T., Beuermann, K., \& Reinsch, K. (San Francisco: ASP), 102

Stockman, H. S., Schmidt, G. D., \& Lamb, D. Q. 1988, ApJ, 332, 282

Tompkin, J., \& Lambert, D. L., 1989, MNRAS, 241, 777

Vacca, W. D., Cushing, M. C., \& Rayner, J. T. 2003, PASP, 115, 389

Walker, M. F. 1965, Mitt. Sternwarte Budapest 57, 1

Wellhouse, J. W., Hoard, D. W., Howell, S. B., Wachter, S., \& Esin, A. A. 2005, 
PASP, in press.

White, J. C., Schlegel, E. M., \& Honeycutt, R. K. 1996, ApJ, 456, 777 


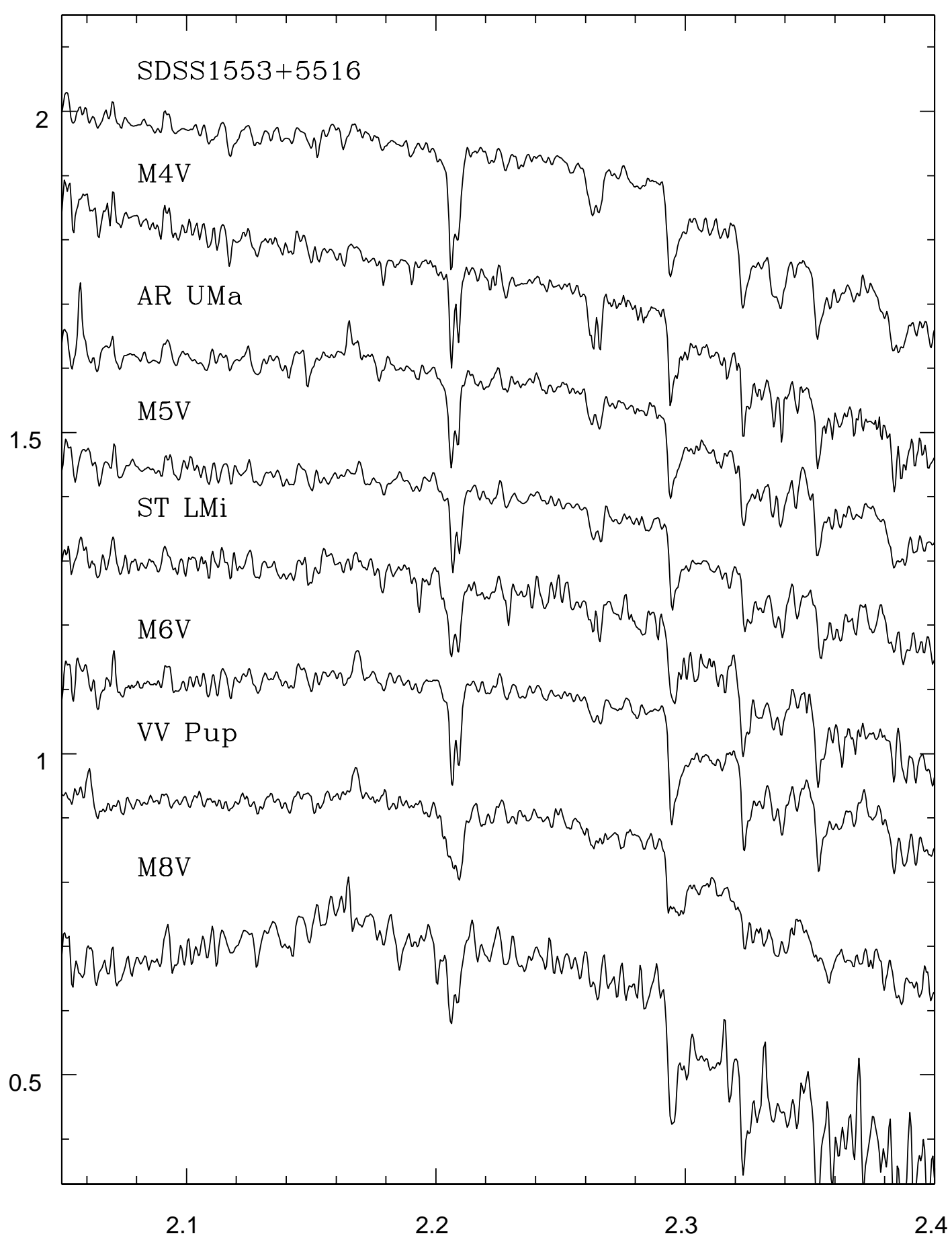

Fig. 1. - The program object spectra compared to the spectra of some late-type dwarfs. To provide a slightly more realistic match to the program object spectra, the data for the M5V, $\mathrm{M} 6 \mathrm{~V}$, and M8V have been rotationally broadened to $150 \mathrm{~km} \mathrm{~s}^{-1}$, while the M4V spectrum 


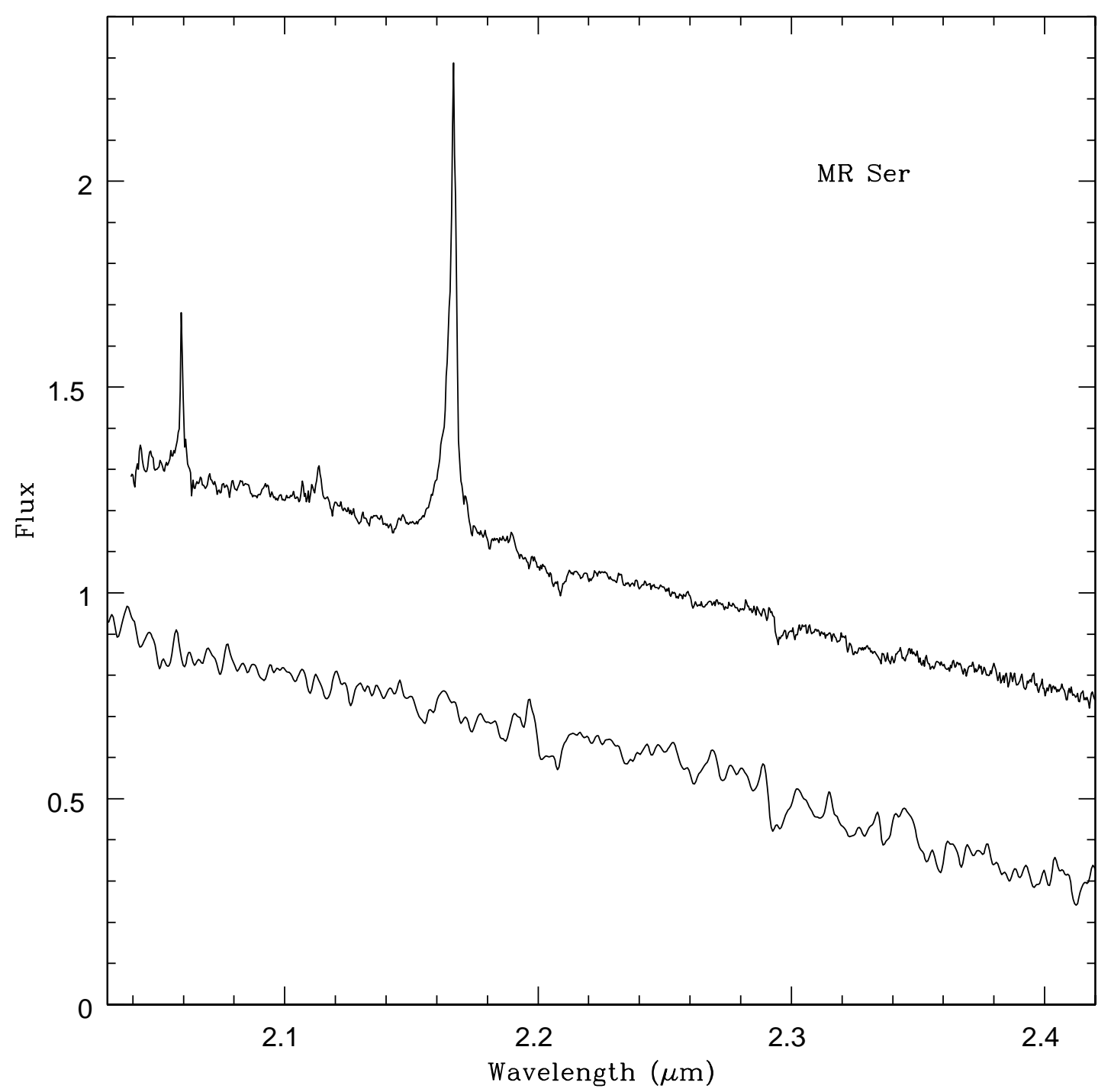

Fig. 2.- The NIRSPEC data for MR Ser (top), compared with the IRTF + SPEX spectrum obtained in 2003 May. Emission lines from He I at 2.06 and $2.11 \mu \mathrm{m}$, and H I Br $\gamma$ at 2.16 $\mu \mathrm{m}$ are present in the NIRSPEC data, but not in the SPEX data. Clearly, MR Ser was in a "high state" in 2005 February. The NIRSPEC data have been Gaussian smoothed to a resolution of $5.1 \AA$ pixel $^{-1}$, while the SPEX data have been smoothed to a resolution of 40 $\AA$ pixel $^{-1}$. 
Table 1. Observation Journal

\begin{tabular}{cccccc}
\hline \hline Object & Porb (hr) & Number of Exps. & Start (UT) & Stop (UT) & Spectral Type \\
\hline VV Pup & 1.674 & 22 & $08: 23$ & $10: 04$ & M7 \\
AR UMa & 1.932 & 6 & $10: 23$ & $10: 50$ & M5.5 \\
ST LMi & 1.898 & 6 & $10: 56$ & $11: 23$ & M6 \\
SDSS J1553 & 4.39 & 12 & $13: 27$ & $14: 24$ & M4.5 \\
MR Ser & 1.891 & 12 & $15: 06$ & $16: 00$ & M8 \\
\hline
\end{tabular}

